\begin{tabular}{lc}
\hline & Sharif University of Technology \\
SCIENTIA & Transactions B: Mechanical Engineering \\
IRAN ICA & www.scientiairanica.com \\
\hline
\end{tabular}

Research Note

\title{
Flexible formation of multiple car-like robots with respect to a dynamic environment
}

\author{
H. Sayyaadi* \\ School of Mechanical Engineering, Sharif University of Technology, Azadi Avenue, Tehran, P.O. Box 11155-9567, Iran.
}

Received 9 September 2013; received in revised form 16 June 2015; accepted 10 August 2015

\author{
KEYWORDS \\ Formation control; \\ Nonholonomic; \\ Car-like mobile \\ robots; \\ Feedback \\ linearization.
}

\begin{abstract}
This paper attempts to give a perspective on decentralized formation control of multiple car-like mobile robots using local information and formation changes in a dynamic environment having several obstacles. In addition, for every mobile robot, it takes physical dimensions, mass, moment of inertia, movement constraints, and saturation of actuators into account. This study makes use of Input/Output Feedback Linearization Method to control each robot. Hence, hierarchical leader-follower based algorithm is employed to control the group formation. To avoid collision between robots and obstacles, and of robots with each other, local artificial potential fields are addressed. The group can change formation with respect to obstacles in the environment. Finally, simulation results of seven individual robots formation are presented to show the performance of the proposed control system.
\end{abstract}

(C) 2016 Sharif University of Technology. All rights reserved.

\section{Introduction}

Formation control of autonomous vehicles has been a well-studied topic in this decade and researcher's attention has been attracted from control of a single mobile robot [1] to control of multiple mobile robots. Advantages that a team of robots can propose are classified as: increasing overall efficiencies due to abilities of any individual robots, robustness against failure and damage, and more systematic approaches to tasks like search and rescue operations [2], automated highways [3], survey and patrols, military missions [4], moving large objects [5], or moving a large number of objects [6].

Formation control mission is control of relative distances and orientation of mobile robots in a group according to the desired patterns for executing a given task [7]. In the literature, several methodologies are available for formation control of mobile agents such

\footnotetext{
*. Tel.: +982166165682; Fax: +98 2166000021

E-mail address: sayyaadi@sharif.edu
}

as Dianwei Qian et al [8]. They used integral sliding mode control and Lyapunov's criteria to form up and maintain robots in predefined trajectories. Dynamic equations of the scheme are subjected to mismatched uncertainties in [8]. Tucker Balch et al. used behaviorbased formation control and their algorithms are experimentally implemented in the real vehicle [9], leaderfollower [10], artificial potential field [11], behaviorbased [12], virtual structures, etc. Perhaps the most popular and intuitive approach is the leader-follower method. One of the advantages of the leader following method is that it is easy to understand and implement. In addition, the formation can be maintained even if the leader is perturbed by some disturbance [13]. In this method, follower robot stays at a specified distance and bearing from a designated leader robot [14], and in the hierarchical leader-follower based formation control, every follower is a leader to the next follower in the chain of robot agents.

Most researchers used kinematic model in the control algorithm of autonomous car-like mobile robots [10]. Kinematic model has its own advantages 
and it helps keeping the steering and velocity of the vehicle entirely decoupled. However, in this process, dynamics of the vehicle are not taken into account and thus this important issue has been remained ignored [4]. Hassan et al. [15] solved the leader-follower control problem for two kinds of mobile robots by using full-state linearization via dynamic feedback; however, they only used kinematic model of mobile robot. Yangmin and Xin [12] used neural network controller to control individual robots, and to control the formation with the use of graph theory. However, they did not study the dynamic effects of the leader robot on the follower. Dierkset and Jagannathan [14] focused on the dynamics of the follower robots as well as the effect of leader's dynamics on the follower (formation dynamics). Hence, they incorporated the formation dynamics in the controller design; however, they did not properly consider collision of robots with each other and with the obstacles in environment. E. Freund and H. Hoyer described an approach to the problem of path planning, including obstacle avoidance in a multi-robot systems with the hierarchical configuration; but the dynamic of every robot and formation dynamic are not addressed properly [16].

A switching formation strategy for multi robots with velocity constraints to avoid and cross obstacles is studied in [17]. In this study, a leader robot plans a safe path using the geometric obstacle avoidance control method. By calculating new desired distances and bearing angles with the leader robot, the follower robots switch into a safe formation.

The autonomous mobile robots considered in this paper are front steer, front drive car-like mobile robots, namely nonholonomic system. In the present study, it is assumed that weight and moment of inertia of wheels of any robot agent are negligible [18], and it is considered that every robot moves on a flat surface in a no-slip wheels condition. Each robot uses local sensory information for position control with respect to a specified leader in the hierarchical configuration, except for the main leader. However, the main leader uses global information for localization and control.

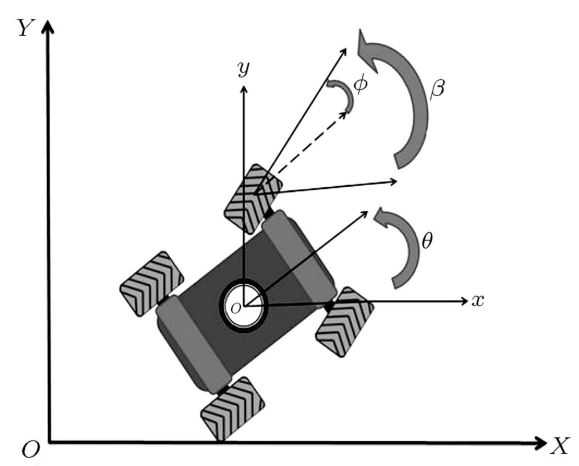

(a)
Local potential field for avoiding collision of robots with each other, and between robots and obstacles, is in use. A pushing force is formed between every robot and obstacle, depending on the distance and relative velocity between them. This force is then converted to desired acceleration for controlling of each robot's motion.

In this article, the proposed method, which is based on dynamic characteristic of the agents and feedback linearization method, is addressed. Control implementation works in the way to control individual robots, while a leader-follower strategy for making the desired formation will be achieved accordingly. Using potential field to avoid collision between agents and obstacles is addressed appropriately.

\section{Mathematical model of car-like mobile robot}

In this study, every robot has two driven wheels (in the rear side) with two steering wheels (in the front side). Based on the theorems of kinematics and dynamics of cars, in order to let them have an instantaneous axis of rotation, the angles of two front wheels have a slight difference while steering. This angle differences do not cause a noticeable discrepancy between the equations of two wheeled and four wheeled robot models. Therefore, four wheeled mobile robot is reduced to bike model [18].

To achieve dynamic model of car-like mobile robot, energy approach is used to derive equations of motion (Lagrangian method). In Lagrangian method, equations of nonholonomic constraints of the mobile robot are needed. Nonholonomic constraints are nonintegralable and are related to the velocity.

In Figure 1, $\theta$ is the body angle, $\phi$ is the steering angle, $c$ is the geometrical center of the robot, $X$ and $Y$ are the symbols of the inertial coordinate system, $F_{1}$ is the moving force of the robot (reaction force of the wheels on the surface), $F_{2}$ is the force exerted on the robot (reaction force on the surface to avoid slippage of front wheels), and $F_{3}$ is the force exerted on the robot from reaction force on the surface to

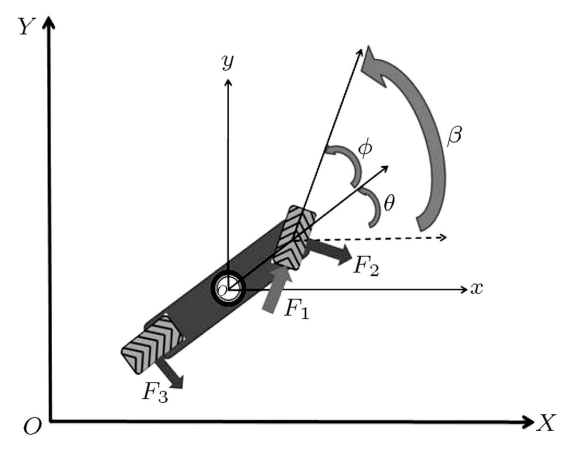

(b)

Figure 1. (a) Schematics of four wheeled car-like robot. (b) The simplified two wheeled one. 
avoid slippage of rear wheels. We assume that the mass and inertia of the wheels are neglected and the slope of the terrain is assumed to be zero. Thus, there is no other source of external forces from the environment. There is no slippage between wheels and the surface. Kinematics equations are derived from the above assumptions and the robot is assumed to be a rigid body. Eq. (1) defines velocity constraints on the rear and front wheels. This means that angular velocity and central linear velocity of the robot should be in agreement with these equations [18].

Nonholonomic constraints are:

$$
\begin{aligned}
& \dot{x} \sin \beta-\dot{y} \cos \beta-d \dot{\theta} \cos \Phi=0, \quad \beta=\theta+\Phi, \\
& \dot{x} \sin \theta-\dot{y} \cos \theta+d \dot{\theta}=0 .
\end{aligned}
$$

Lagrange equations for nonholonomic systems with a dependent set of generalized coordinates $q_{i}$ 's are [18]:

$$
\frac{d}{d t}\left(\frac{\partial L}{\partial \dot{q}_{i}}\right)-\frac{\partial L}{\partial q_{i}}=Q_{i}^{\prime}+\sum_{j=1}^{m} \lambda_{j} a_{i j}, \quad(i=1,2, \cdots, n) .
$$

in Eq. (2), $a_{i j}(q, t)=\frac{\partial g_{j}}{\partial q_{i}}$, where $g_{j}$ 's are equations of nonholonomic constraints and $L(q, \dot{q}, t)=T(q, \dot{q}, t)-$ $V(q, \dot{q}, t)$ is Lagrangian function. We suppose that $\tau_{1}^{\prime}$ and $\tau_{2}^{\prime}$ are the main torques of steering and front wheels, and thus:

$$
F_{1}=\frac{\tau_{1}^{\prime}}{r_{w}}=\tau_{1}, \quad \text { and } \quad \dot{\phi}=C_{s} \tau_{2}^{\prime}=\tau_{2},
$$

where $r_{w}$ is the radius of the wheels and $C_{s}$ is the damping factor of the steering. In order to simplify these equations, the inputs are chosen as $F_{1}=\tau_{1}$ and $\dot{\phi}=\tau_{2}$; finally, by multiplying and dividing the results by $r_{w}$ and $C_{s}$, the main torques are derived. The effect of steering damping is more than that of the inertia; therefore, in the equation of steering torques, damping is considered to be dominant and thus, inertia is negligible. From Eqs. (1) and (2), the governing equations of the robot can be written in state space form as follows. Derivations of $w, f_{i}$, and $g_{i j}$ are brought in the Appendix.

$$
\begin{aligned}
& \dot{X}=f(X)+G(X) . U, \quad Y=h(X), \\
& X=\left[\begin{array}{lllllll}
x & \dot{x} & y & \dot{y} & \theta & \dot{\theta} & \phi
\end{array}\right]^{T}, \\
& f(X)=\left[\begin{array}{lllllll}
\dot{x} & f_{2} & \dot{y} & f_{4} & \dot{\theta} & f_{6} & 0
\end{array}\right]^{T}, \\
& G(X)=\left[\begin{array}{cc}
0 & 0 \\
g_{21} & g_{22} \\
0 & 0 \\
g_{41} & g_{42} \\
0 & 0 \\
g_{61} & g_{62} \\
0 & 1
\end{array}\right], \quad U=\left[\begin{array}{l}
\tau_{1} \\
\tau_{2}
\end{array}\right], \quad h(X)=\left[\begin{array}{l}
x \\
y
\end{array}\right]
\end{aligned}
$$

\section{Control of each car-like mobile robot individually}

Since all of the robots used in the formation are the same, in this section, a controller is designed for each individual robot. For this purpose, we used feedback linearization. Considering $Y=(x, y)$ as the output and $U=\left(\tau_{1}, \tau_{2}\right)$ as the input, the first step is to find the relative degree of the system. Using Eq. (5) represented by [20], System (3) will have a relative degree of $r_{i}$ if the following equation is satisfied for at least one $j$ :

$$
\begin{aligned}
& L_{g_{j}} L_{f}^{a_{i}} h_{i}(x)=0, \quad 0<a_{i}<r_{i}-1, \\
& L_{g_{j}} L_{f}^{r_{i}-1} h_{i}(x) \neq 0 .
\end{aligned}
$$

Using Eq. (5), the system defined by state Eq. (3) has a relative degree of $(2,2)$, and the relative degree of the whole system is $r=4$.

Using Eq. (6) from [20] and knowing $r_{i}$ for each output $y_{i}$, Eq. (7) will be obtained:

$$
\begin{aligned}
& y_{i}^{\left(r_{i}\right)}=L_{f}^{r_{i}} h_{i}+\sum_{j=1}^{m} L_{g_{j}} L_{f}^{r_{i}-1} h_{i} u_{i}, \\
& \left(\begin{array}{l}
\ddot{x} \\
\ddot{y}
\end{array}\right)=\left(\begin{array}{l}
f_{2} \\
f_{4}
\end{array}\right)+E(X) U, \\
& E(X)=w \cdot\left[\begin{array}{ll}
g_{21} & g_{22} \\
g_{41} & g_{42}
\end{array}\right] .
\end{aligned}
$$

Variables $f_{i}, g_{i j}$, and $w$ are shown in the Appendix. Necessary condition for input-output linearization is that the inverse of decoupling matrix $E$ exists. Considering the derived equations, the decoupling matrix has the full rank, except for the condition that the speed of center of the robot is zero, or say it does not move. For the sake of feedback linearization, when the decoupling matrix is singular, it is possible to use methods of dynamic extension (redefining inputs) or system inversion (redefining outputs). Here, we use a more straightforward method to solve the problem.

We put a constraint on the velocity so that if the linear velocity of the center of robot $v=\sqrt{\dot{x}^{2}+\dot{y}^{2}}$ descends below a minimum threshold, then we take the velocity back to the threshold. Thus, if $|\dot{x}|<$ $\dot{x}_{\text {threshold }}$ and $|\dot{y}|<\dot{y}_{\text {threshold, }}$, we will have $|\dot{x}|=$ $\operatorname{sign}(\dot{x}) \dot{x}_{\text {threshold }}[1]$.

We use an artificial input $V$ as a linear pole placement controller as follows:

$$
V=\left(\begin{array}{l}
\ddot{x}_{d}-k_{1}\left(\dot{x}-\dot{x}_{d}\right)-k_{2}\left(x-x_{d}\right) \\
\ddot{y}_{d}-k_{3}\left(\dot{y}-\dot{y}_{d}\right)-k_{4}\left(y-y_{d}\right)
\end{array}\right),
$$

where $k_{i}$ 's are chosen so that all of the poles of polynomials (Eq. (9)) are on the left side of the imaginary 
axis [1]:

$$
\begin{aligned}
& P_{1}(s)=s^{2}+k_{1} s+k_{2}, \\
& P_{2}(s)=s^{2}+k_{3} s+k_{4} .
\end{aligned}
$$

Thus, inputs of the controller can be found as follows:

$$
U=E^{-1}\left[\begin{array}{l}
\ddot{x}_{d}-k_{1}\left(\dot{x}-\dot{x}_{d}\right)-k_{2}\left(x-x_{d}\right)-f_{2} \\
\ddot{y}_{d}-k_{3}\left(\dot{y}-\dot{y}_{d}\right)-k_{4}\left(y-y_{d}\right)-f_{4}
\end{array}\right] .
$$

In Eq. (10), $\left(x_{d}, y_{d}\right)$ is the desired position, $\left(\dot{x}_{d}, \dot{y}_{d}\right)$ is the desired velocity, and $\left(\ddot{x}_{d}, \ddot{y}_{d}\right)$ is the desired acceleration of the center of the robot. These are used in the next section to control the motion of robots in order to achieve a desired formation.

The decoupling control rule (Eq. (10)) will result in simple linear equation (Eq. (11)), in which input $V_{i}$ only affects output $Y_{i}$.

$$
\ddot{Y}=V \text {. }
$$

\section{Governing assumptions about constraints and saturation of actuators}

The actuators of the robots also include torque saturation as follows:

$$
\left|\tau_{1}\right| \leq \tau_{1_{\max }}, \quad\left|\tau_{2}\right| \leq \tau_{2_{\max }} .
$$

$\tau_{1_{\max }}$ and $\tau_{2_{\max }}$ are respectively the maximum absolute driving torque and steering torque that actuators can apply to the model. Torque scaling is proposed in (13). If the scaled driving torque and steering torque are $\tau_{1 \mathrm{~s}}$ and $\tau_{2 s}$, respectively, we will have [21]:

$$
\begin{aligned}
& \rho=\max \left\{\left|\tau_{1}\right| / \tau_{1 \max }, \quad\left|\tau_{1}\right| / \tau_{1 \max }, \quad 1\right\}, \\
& \tau_{1 s}=\operatorname{sign}\left(\tau_{1}\right) \tau_{1 \max }, \quad \tau_{2 s}=\frac{\tau_{2}}{\rho}, \\
& \text { when } \quad \rho==\frac{\left|\tau_{1}\right|}{\tau_{1 \max }}, \\
& \tau_{1 s}=\frac{\tau_{1}}{\rho}, \quad \tau_{2 s}=\operatorname{sign}\left(\tau_{2}\right) \tau_{2 \max }, \\
& \text { when } \quad \rho==\frac{\left|\tau_{2}\right|}{\tau_{2 \max }}, \\
& \tau_{1 s}=\tau_{1}, \quad \tau_{2 s}=\tau_{2}, \quad \text { when } \quad \rho==1 .
\end{aligned}
$$

With increasing the frequency of the desired input signal, physical actuators cannot exert desired control torques. Eq. (14) states that a real actuator is unable to reconstruct a control signal with high frequency:

$$
\tau_{f}(s)=\frac{\tau(s)}{\rho s+1} .
$$

$\tau(s)$ is the ideal control torque signal and $\tau_{f}(s)$ is the output of the real actuator. In Eq. (14), $\rho$ is the inverse of the filter pole and increasing it will reduce the ability of the system to follow a step input. Eq. (15) shows the discrete form of the filter [2]:

$$
\tau_{f}(t+1)=\tau_{f}(t) e^{\frac{-d t}{\rho}}+\left(\tau(t+1)+\tau(t) e^{-\frac{d t}{\rho}}\right) \frac{d t}{2 \rho} .
$$

In accordance with the dynamics of actual vehicles, when using control algorithm (Eq. (10)), steering angle can change in the range of $|\phi|<\phi_{\max }$. For this, we consider a constraint for steering angle in the calculations. Eq. (16) shows the discrete form of this constraint:

$$
\begin{aligned}
& d X_{i}(t+1)=\left\{\begin{array}{lll}
d X_{i}(t+1) & \text { when } & |\phi|<\phi_{\max } \\
d X_{i}(t) & \text { when } & |\phi| \geq \phi_{\max }
\end{array}\right. \\
& X_{i}(t+1)=X_{i}(t)+d X_{i}(t+1) d t, \\
& \phi(t+1)=\left\{\begin{array}{lll}
\phi(t+1) & \text { when } & |\phi|<\phi_{\max } \\
\phi(t) & \text { when } & |\phi| \geq \phi_{\max }
\end{array}\right.
\end{aligned}
$$

In Eq. (16), $d X_{i}$ is the change in the state variables in a period of $d t$ and $X_{i}$ 's are the state variables of the system.

In the presence of nonholonomic constraints in a car-like robot, we have the kinematic (Eqs. (17)) for each robot:

$$
\begin{aligned}
& \dot{x}=v(2 \cos \phi \cos \theta-\sin \phi \sin \theta), \\
& \dot{y}=v(2 \sin \theta \cos \phi+\cos \theta \sin \phi), \\
& \dot{\theta}=\frac{v \sin \phi}{d} .
\end{aligned}
$$

Considering the kinematic equations (Eqs. (17)), knowing the body angle, steering angle, and front wheel velocity, we can determine $\dot{\theta}$ and $(\dot{x}, \dot{y})$. Considering that the control inputs are steering torque and front wheel torque, it can be assumed that the robot is able to change $\phi$ and $v$ to the values that are needed to achieve the desired center position. However, determination of the desired center positions $\left(x_{d}, y_{d}\right)$ for each $v$ and $\phi$ is not feasible due to dependence of equations on body angle $\theta$. Consequently, the robot cannot choose an arbitrary path. This problem will worsen as we take into account the effects of constraints on steering angle $|\phi|<\phi_{\max }$, mass and moment of inertia of the robot, saturation of actuators, and steering damping.

\section{Design of the controller for the group formation}

Local formation control means controlling the relative position and orientation of the agents, with respect to 


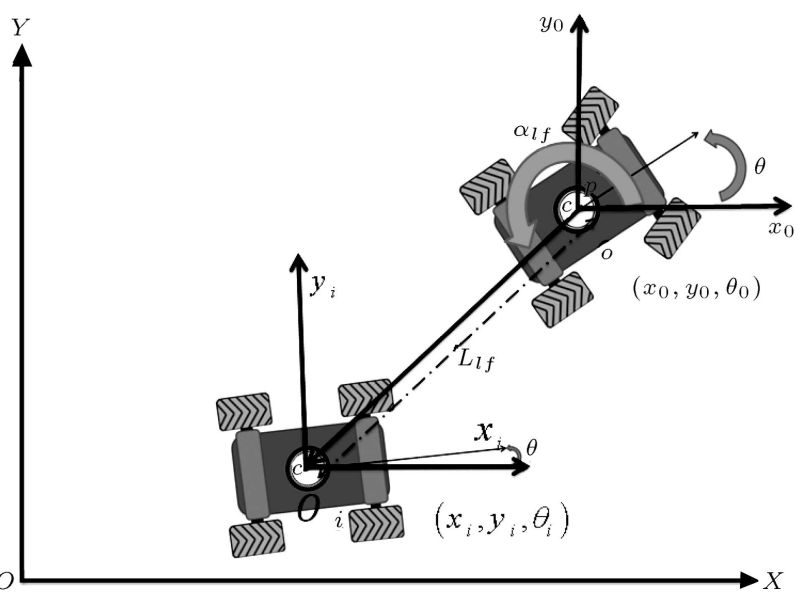

Figure 2. Distance and bearing of follower robot with respect to leader.

other agents according to some desired pattern, for executing a given task. We used separation-bearing $(L, \alpha)$ technique to create the desired formation for the group. Referring to Figure 2, the separation distance and bearing can be taken as:

$$
L_{L f}=\sqrt{x_{i}^{2}+y_{i}^{2}}, \quad \alpha_{L f}=\tan ^{-1}\left(\frac{y_{i}}{x_{i}}\right),
$$

where $\mu_{i}=\left(x_{i}, y_{i}\right)$ is the position of follower with respect to the local coordinate system that is attached to the leader. Position states of follower are computed locally with respect to the leader and other global states are achieved with the use of local sensors.

In controller design of the car-like mobile robot, the relation between the states and torque is defined. In this section, desired position $\mu_{d_{i}}=\left(x_{d_{i}}, y_{d_{i}}\right)$, velocity, and acceleration of the follower $i$ would be calculated with respect to the local coordinate system which is attached to its leader. To control the formation, we consider two kinds of generic formations: rigid formation and convey-like formation [7].

\subsection{Rigid formation}

If the motion of each group member is proportional to the displacement or rotation of the formation, the formation is considered solid. This is as each member is part of a solid body. In this situation, the leader follows a predefined path and other robots keep a distance and angle from the leader in the hierarchical pattern. Using Eq. (19), we can determine the desired local position $\mu_{d_{i}}$, desired velocity, $\dot{\mu}_{d_{i}}$, and desired acceleration, $\ddot{\mu}_{d_{i}}$ of each robot:

$$
\begin{aligned}
& \xi_{d_{2}}=\mu_{d_{2}}+\xi_{1}, \\
& \xi_{d_{3}}=\mu_{d_{3}}+\xi_{1}, \\
& \xi_{d_{(2 i-1)}}=\mu_{d_{(2 i-1)}}+\xi_{(2(i-1)-1)}, \quad i=3, \cdots, N / 2, \\
& \xi_{d_{2 i}}=\mu_{d_{2 i}}+\xi_{2(i-1)}, \quad i=2, \cdots, N / 2,
\end{aligned}
$$

$$
\begin{aligned}
& \mu_{d_{i}}=L_{i}^{z}\left[\begin{array}{c}
\cos \alpha_{i}^{z} \\
\sin \alpha_{i}^{z}
\end{array}\right], \quad z=[1,2], \\
& \dot{\mu}_{d_{i}}=\dot{\mu}_{1}, \quad \ddot{\mu}_{d_{i}}=\ddot{\xi}_{1}+\ddot{\Lambda}_{d_{i}} .
\end{aligned}
$$

In Eq. (19), $\xi_{i}$ is the global position of every robot and is only used to clarify the equations, $N$ is the number of agents, and $z$ is the shape of the formation. $\ddot{\Lambda}_{d_{i}}$ would be calculated to avoid collision between robots, and between robots and dynamic obstacles. There is an appropriate and simple algorithm which is consistent with intuitive point of view and dynamical analysis. If the control of the group of inertial agents is not done properly, it may lead to chaos in the group. As a result, it is necessary to understand the dynamic behavior of the agents [19].

\subsection{Convey-like formation}

Assuming a desired motion path for the leader, Control equation (Eq. (10)) determines the control torques for the leader. Applying this torques to the model, linear and angular velocities and accelerations of the leader robot are determined. Now, using Eq. (20), we can get the desired position, velocities, and accelerations of each follower robot with respect to its leader in a hierarchical manner:

$$
\begin{aligned}
& \xi_{d_{i}}=\eta_{d_{i}}+\xi_{i-1}, \quad i=2, \cdots, N, \\
& \eta_{d_{i}}=L_{i}^{3}\left[\begin{array}{c}
\cos \theta_{i-1} \\
\sin \theta_{i-1}
\end{array}\right], \\
& \dot{\eta}_{d}=L_{i}^{3} \dot{\theta}_{i-1}\left[\begin{array}{c}
-\sin \theta_{i-1} \\
\cos \theta_{i-1}
\end{array}\right], \\
& \dot{\xi}_{d_{i}}=\dot{\eta}_{d_{i}}+\dot{\xi}_{i-1}, \\
& \ddot{\xi}_{d_{i}}=\ddot{\xi}_{i-1}+\ddot{\Lambda}_{d_{i}} .
\end{aligned}
$$

In Eq. (20), $\theta_{i}$ is the body angle of the $i$ th agent, and $\ddot{\Lambda}_{d_{i}}$ is the desired acceleration of each robot, which will be computed in the next section.

\section{Collision avoidance and obstacle avoidance}

In this section, we use a dummy spring and damper to achieve the desired acceleration of the $i$ th robot $\ddot{\Lambda}_{d_{i}}$. In Figure $3, k_{s}$ is the elasticity constant and $C_{L}$ is the damping coefficient. Eq. (21) shows the desired acceleration of the $i$ th robot, where $R_{j}$ is the relative distance between robot and the $j$ th agent and $R_{R}$ is the equilibrium distance between them; $R_{k}$ is the distance between the robots and the $k$ th obstacle; $R_{o}$ is the equilibrium distance between them, $k_{p}$, and $k_{p_{o}}$ are elasticity coefficients to adjust the distances between one robot and other agents and obstacle, respectively; $k_{d}$, and $k_{d_{o}}$ are damping coefficients to reduce the 


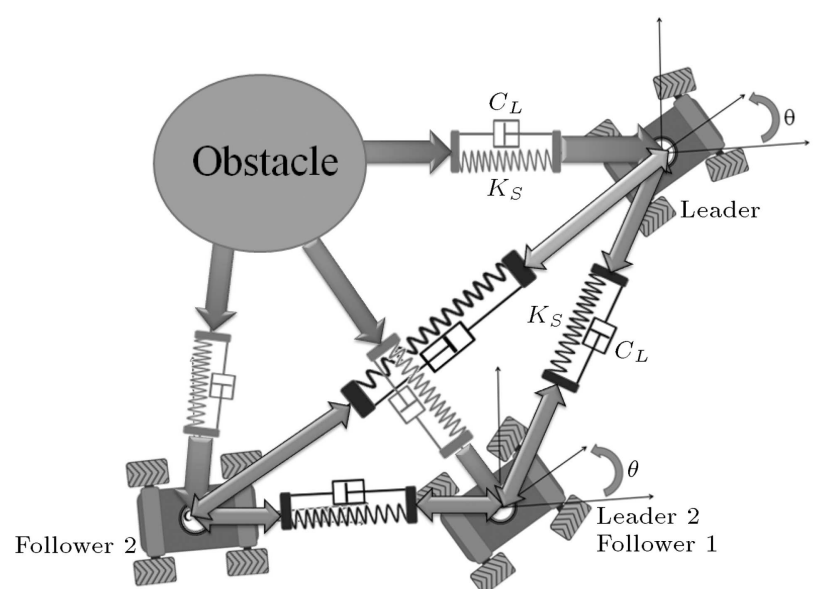

Figure 3. Structure of spring and damper connection between agents and obstacle.

relative speed between the robot and other agents and obstacles, respectively; finally, $n$ and $m$ are the numbers of robots and obstacles, respectively:

$$
\begin{aligned}
& \ddot{\Lambda}_{d_{i}}=\ddot{\Lambda}_{\text {agents }}+\ddot{\Lambda}_{\text {obstacles }}+\ddot{\Lambda}_{\text {leader }}, \\
& \ddot{\Lambda}_{\text {agents }}=\frac{1}{m} \sum_{\substack{j=1 \\
j>i}}^{n}\left(\left(\frac{k_{p}}{R_{j}\left(R_{j}-R_{R}\right)}\right)\left[\begin{array}{l}
\left(x_{j}-x_{i}\right) \\
\left(y_{j}-y_{i}\right)
\end{array}\right]\right. \\
& \left.+k_{d}\left[\begin{array}{l}
\left(\dot{x}_{j}-\dot{x}_{i}\right) \\
\left(\dot{y}_{j}-\dot{y}_{i}\right)
\end{array}\right]\right) \\
& \ddot{\Lambda}_{\text {obstacles }}=\frac{1}{m} \sum_{k=1}^{m}\left(\left(\frac{k_{p_{o}}}{R_{k}\left(R_{k}-R_{o}\right)}\right)\left[\begin{array}{l}
\left(x_{i}-x_{k}\right) \\
\left(y_{i}-y_{k}\right)
\end{array}\right]\right. \\
& \left.\quad+k_{d_{o}}\left[\begin{array}{l}
\left(\dot{x}_{k}-\dot{x}_{i}\right) \\
\left(\dot{y}_{k}-\dot{y}_{i}\right)
\end{array}\right]\right) \\
& \ddot{\Lambda}_{\text {leader }}=\left[\begin{array}{l}
\ddot{x}_{L} \\
\ddot{y}_{L}
\end{array}\right] .
\end{aligned}
$$

In Eq. (21), first term prevents collision between the agents, $\ddot{\Lambda}_{\text {obstacles }}$ is for collision avoidance between robots and obstacles, and $\ddot{\Lambda}_{\text {leader }}$ is the acceleration of leader of the agent and makes the robot track the leader properly.

\section{Selecting desired formation for the group}

To change the formation, each robot calculates the repulsive force generated from obstacles and shares it with other robots. Total repulsive force is equal to the summation of all absolute virtual forces from obstacles on each agent. If this value exceeds a maximum value, the group will change its formation from an arrow to a double-column and if it exceeds a second maximum, the formation will change to a convoy-like one. After passing the obstacles, the repulsive force decreases and the formation changes vice versa.

\section{Simulation results}

We carried out a numerical simulation to illustrate the dynamic performance of the controller. An arrowhead formation of seven nonholonomic car-like mobile robots is considered where the main leader follows a predefined desired trajectory. When agents approach obstacles, they change their formation to a double-line and convoy-like formation. Simulations are carried out in MATLAB. Parameters used in this section are shown in Table 1.

In Figure 4, the group is successfully tracking its desired path and the vehicles reach their desired formation configuration. As shown in this figure, as robots approach the obstacles, they change their formation with respect to obstacles.

According to the graphs in Figure 4, the desired path of the group leader is defined such that the robots collide an obstacle at $t=16.8(\mathrm{~s})$. However, because of the virtual repulsive force generated from the obstacle, the group pass it without collision and follow the desired path with some distance. These graphs in Figure 4 clearly indicate that when the group are passing through the obstacles, they can change shape of the formation with respect to the obstacles.

In Figure 5, desired acceleration of agents with respect to time is shown. As shown in this figure, when the agents approach an obstacle, through virtual repulsive force of obstacle and other agents, the desired accelerations in the directions of axes $x$ and $y$ rise; but when the group pass the obstacles, desired accelerations converge to zero and it means the robots move freely to the desired position.

Figure 6 shows the relative distance and angle errors of the follower robots with respect to the desired values in the leader's coordinate system. It is seen that with the change of formation, error values increase sharply; but as the formation becomes stable, these errors converge to zero and the group achieve the predefined formation. This figure clearly indicates that the robots have arrived the desired position quickly without overshot and undershot.

Figure 7 shows the body and steering angles of agents in the formation. This figure clearly indicates that all of the agents move forward (because the body angle is lower than 90 degrees) in the same direction and the steering angles are under that saturation angle (60 degrees considered to be more realistic).

Figure 8 shows the steering torques and driving torques. We assume that every robot moves in a frictionless environment with constant velocity in $x$ 
Table 1. Geometric, physical, and control parameters used in simulations.

\begin{tabular}{|c|c|}
\hline Parameter & Description \\
\hline$d t=0.05(\mathrm{~s})$ & Step time for discretization \\
\hline $\begin{array}{l}d=0.3(\mathrm{~m}) \\
w=0.2(\mathrm{~m})\end{array}$ & Length and width of robot \\
\hline$M=6(\mathrm{~kg})$ & Mass of robot \\
\hline$I_{c}=0.065\left(\mathrm{~kg} \cdot \mathrm{m}^{2}\right)$ & Moment of inertia with respect to center of gravity \\
\hline $\begin{array}{l}\tau_{1 \max }=1(\mathrm{~N} . \mathrm{m}) \\
\tau_{2 \max }=1(\mathrm{~N} . \mathrm{m})\end{array}$ & Maximum torque of actuators \\
\hline$\phi_{1 \max }=60(\mathrm{deg})$ & Saturation angle of steering \\
\hline$L_{i}^{1}=1.6(\mathrm{~m})$ & Separation distance between robots in arrowhead formation \\
\hline $\begin{array}{l}\alpha_{i}^{1}=135(\mathrm{deg}) \\
i=2 n \\
n=1, \cdots, N / 2\end{array}$ & Separation bearing between even robots in arrowhead formation \\
\hline $\begin{array}{l}\alpha_{i}^{1}=225(\mathrm{deg}) \\
i=(2 n-1) \\
n=1, \cdots, N / 2\end{array}$ & Separation bearing between odd robots in arrowhead formation \\
\hline $\begin{array}{l}L_{2}^{2}=L_{3}^{2}=1(\mathrm{~m}) \\
L_{i}^{2}=1.6(\mathrm{~m}) i \neq 2,3\end{array}$ & Separation distance between robots in two-line formation \\
\hline $\begin{array}{l}\alpha_{2}^{2}=135(\mathrm{deg}) \\
\alpha_{3}^{2}=225(\mathrm{deg}) \\
\alpha_{i}^{2}=0(\mathrm{deg}) i \neq 2,3\end{array}$ & Separation bearing between robots in two-line formation \\
\hline$L_{i}^{3}=1(\mathrm{~m})$ & Separation distance between robots in convoy-like formation \\
\hline$F_{1 c}=60(\mathrm{~N})$ & $\begin{array}{l}\text { Maximum allowable force in order to change from arrow formation } \\
\text { to double-line formation }\end{array}$ \\
\hline$F_{2 c}=150(\mathrm{~N})$ & $\begin{array}{l}\text { Maximum allowable force in order to change from double-line } \\
\text { formation to convoy-like formation }\end{array}$ \\
\hline$R_{R}=0.1(\mathrm{~m})$ & Equilibrium distance between robots (minimum allowable distance) \\
\hline$R_{o}=0.2(\mathrm{~m})$ & $\begin{array}{l}\text { Equilibrium distance between robots and obstacles (minimum } \\
\text { allowable distance) }\end{array}$ \\
\hline $\begin{array}{l}K_{1}=20 \\
K_{2}=5\end{array}$ & Coefficient of controller in $x$ direction \\
\hline $\begin{array}{l}K_{3}=25 \\
K_{4}=6\end{array}$ & Coefficient of controller in $y$ direction \\
\hline$C=0.5$ & Coefficient of steering damper \\
\hline $\begin{array}{l}K_{p}^{o b}=0.04 \\
K_{d}^{o b}=0.04\end{array}$ & Damping and spring coefficients between robots and obstacles \\
\hline $\begin{array}{l}K_{p}=3 \\
K_{d}=26\end{array}$ & Damping and spring (repulsive) coefficients between robots \\
\hline
\end{tabular}



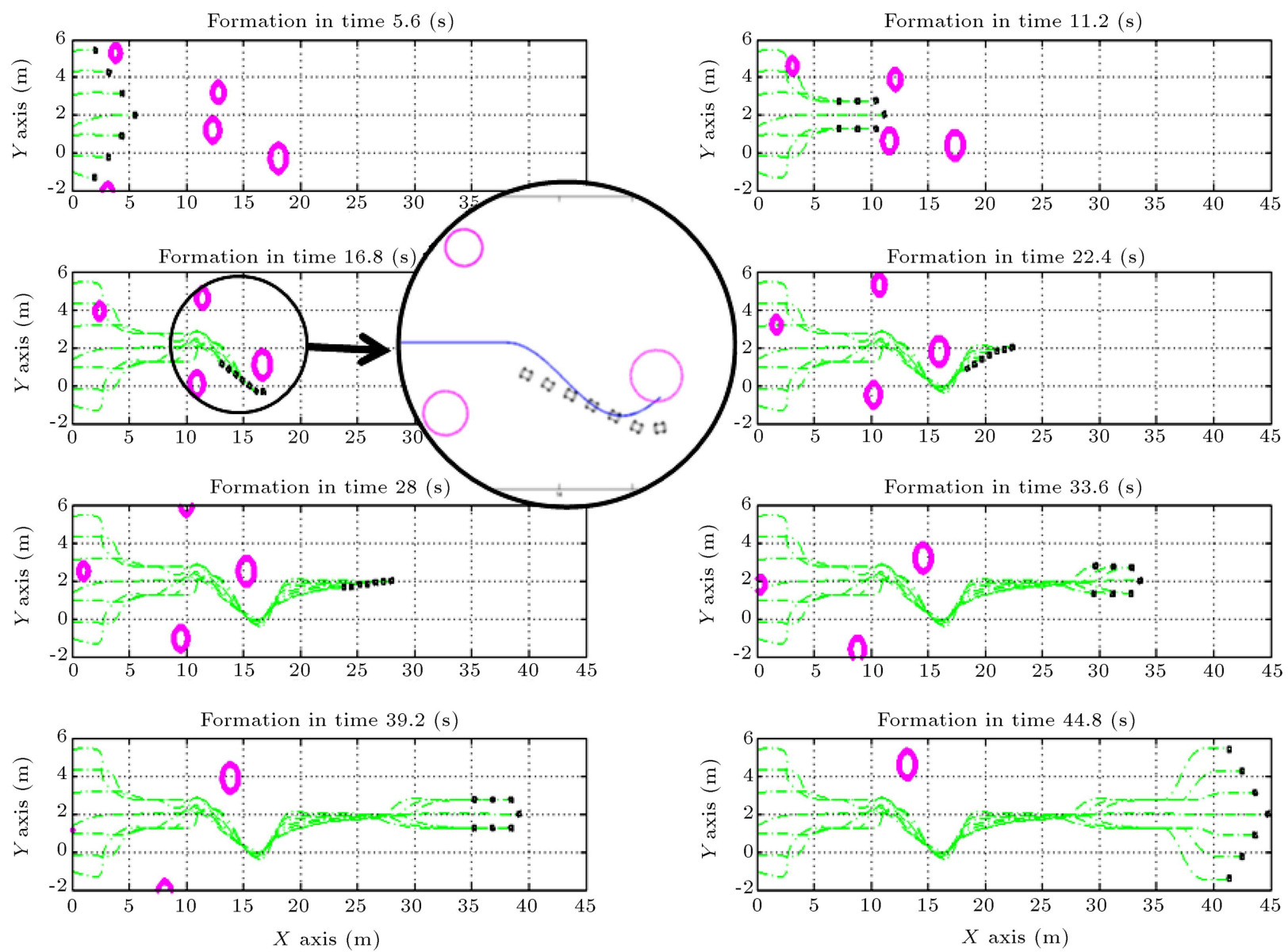

Figure 4. Change in formation of seven nonholonomic car-like mobile robots with respect to obstacles.
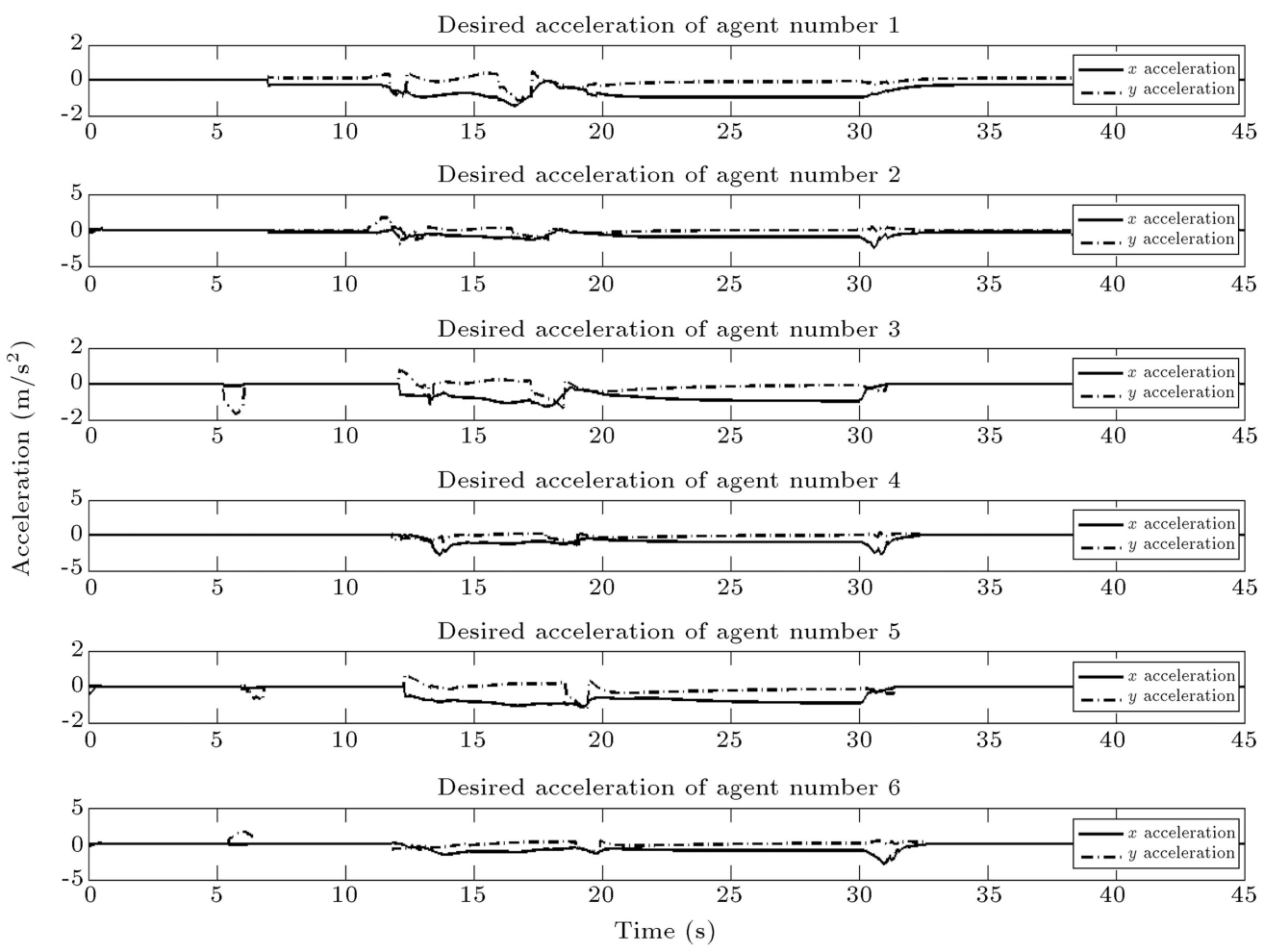

Figure 5. Desired acceleration of agents. 

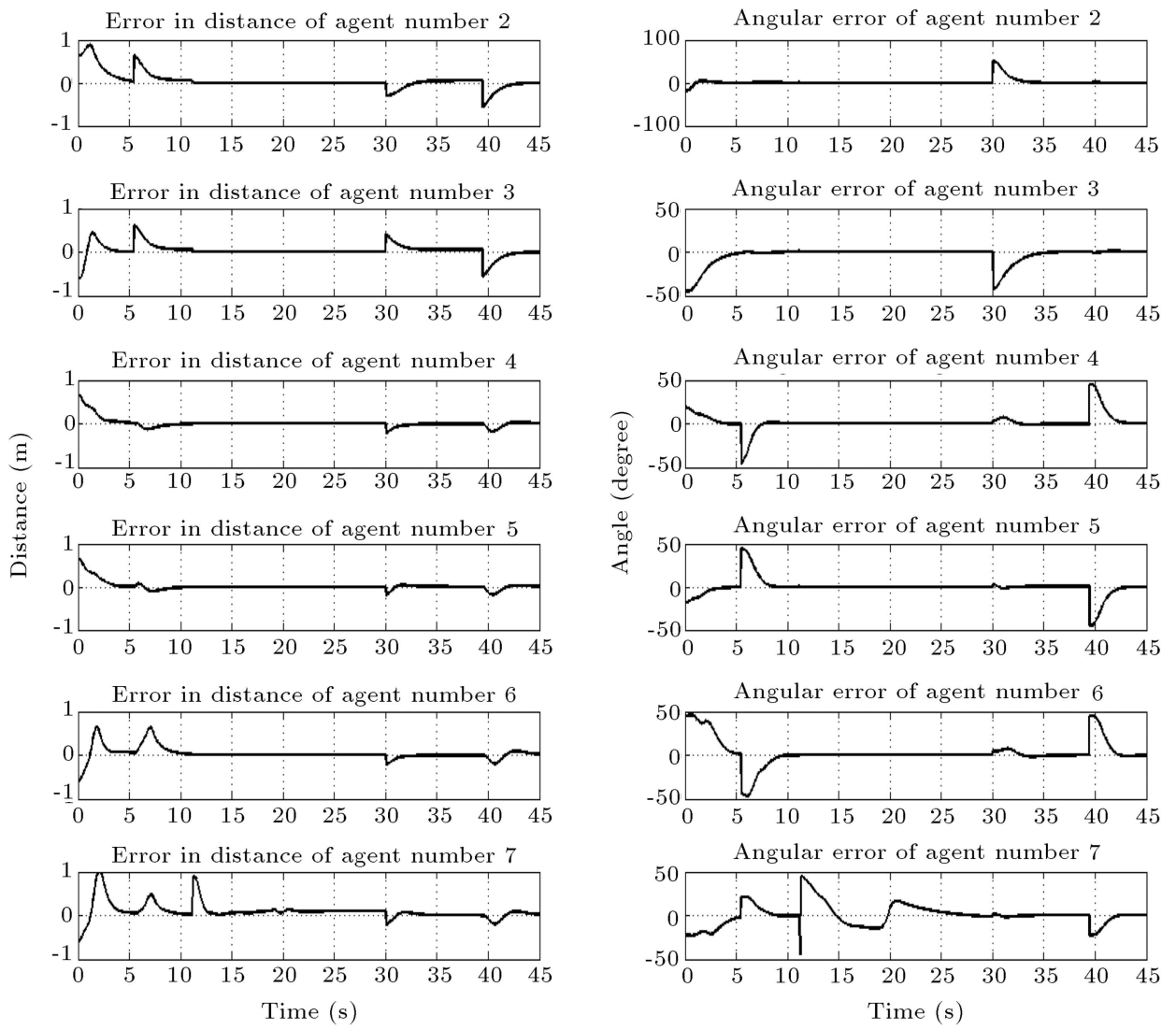

Figure 6. Relative distance and angular errors of robots.
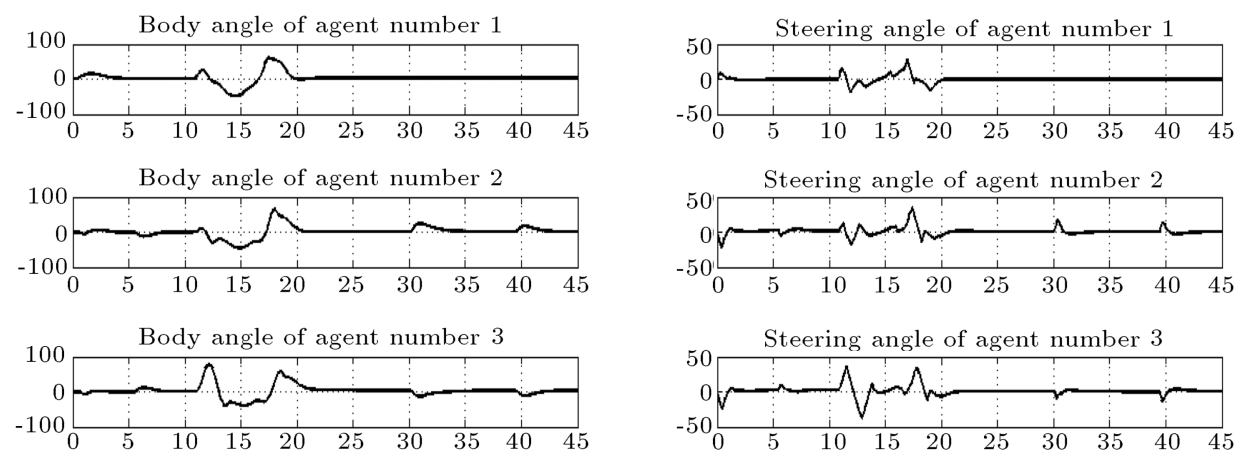

Body angle of agent number 4

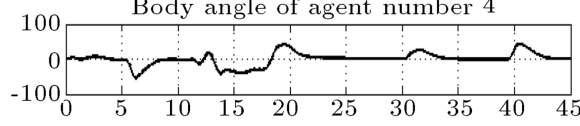

Body angle of agent number 5
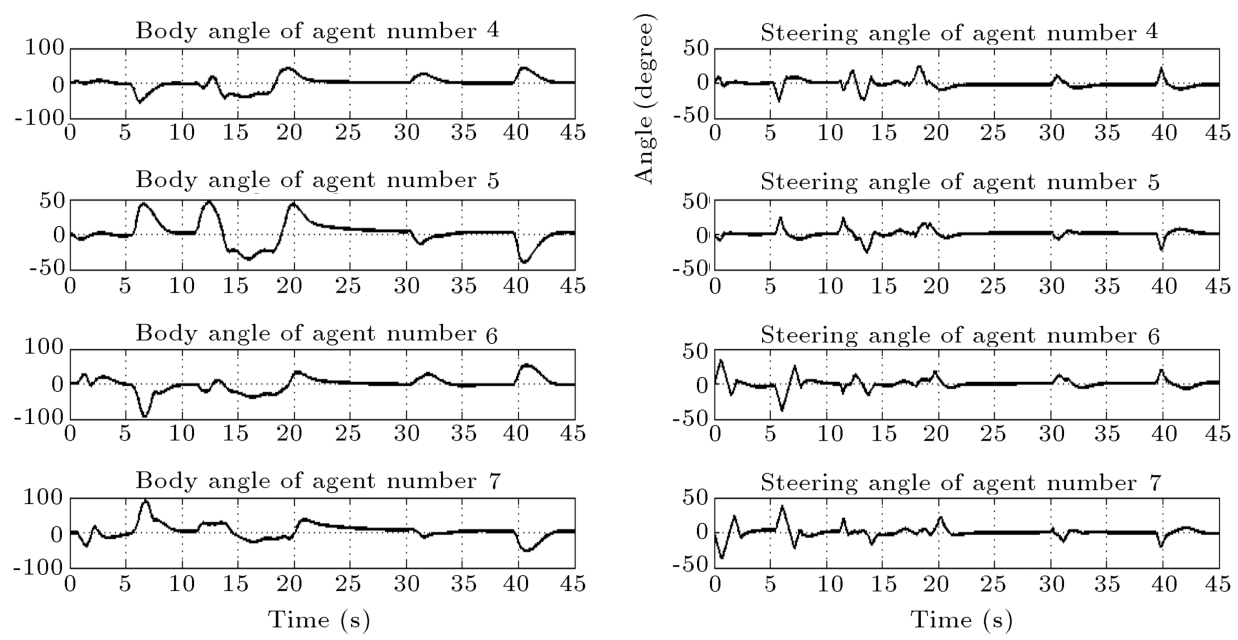

Figure 7. Body and steering angles of agents in the formation. 

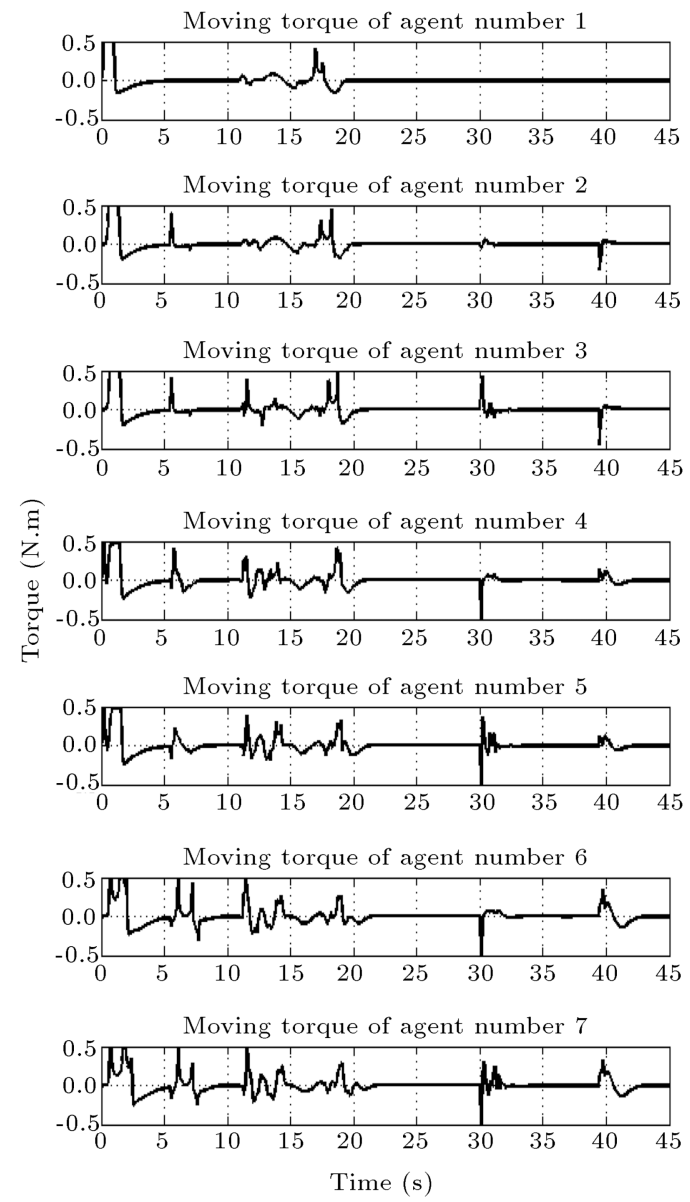
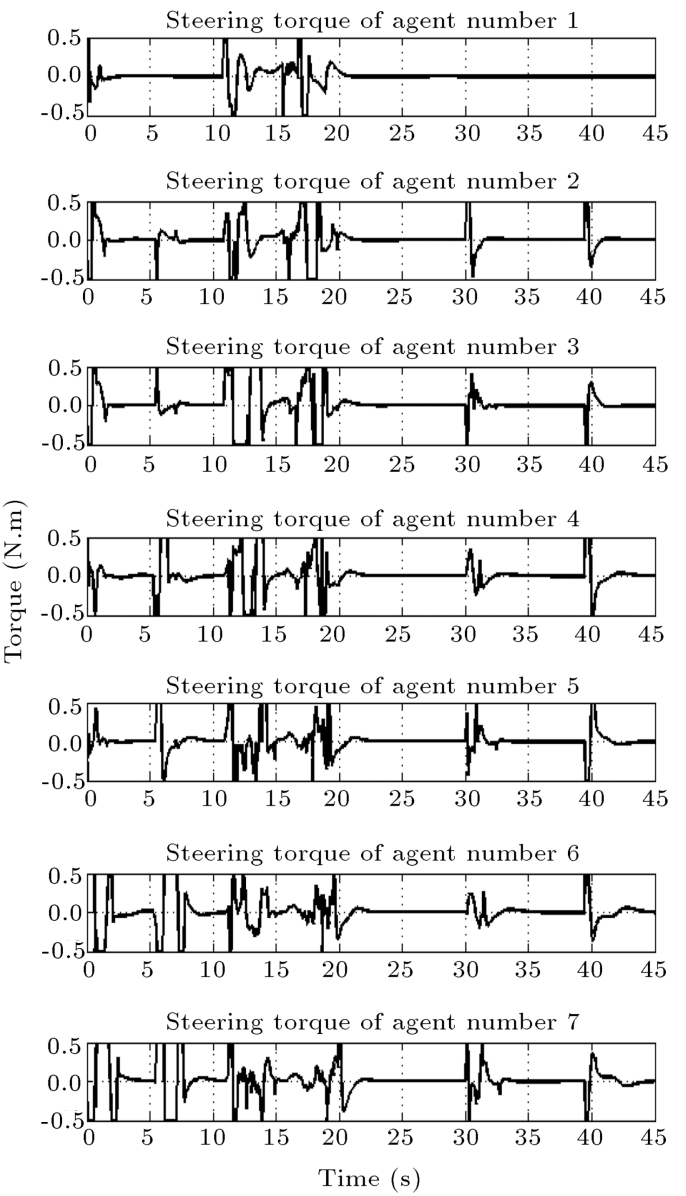

Figure 8. Steering torques and driving torques.

direction; thus, after they pass the obstacles and achieve the desired formation, steering and driving torques converge to zero. As this figure shows, control torques reach saturation limits several times during the simulation.

\section{Conclusion}

In the present study, formation control of multiple carlike mobile robots in a dynamic environment with local information and formation changes in the presence of obstacles was investigated. The approach uses input/output feedback linearization and local artificial potential fields for avoiding collision among robots, and between robots and obstacles. By understanding the dynamic behavior of robots, a more comprehensible algorithm is proposed which requires minimal calculations and is capable of controlling the formation of robots in a decentralized and scalable manner. Simulation results show the effectiveness of the proposed method. Future work can include the design of state observers for each robot to reduce the number of required sensors, analysis robustness of the system against sensor failures, or insufficient data transmission due to communication problems.

\section{References}

1. Egerstedt, M., Hu, X. and Stotsky, A. "Control of mobile platforms using a virtual vehicle approach", Automatic Control, IEEE Transactions on, 46, pp. 1777-1782 (2001).

2. Murphy, R.R. "Human-robot interaction in rescue robotics", Systems, Man, and Cybernetics, Part C: Applications and Reviews, IEEE Transactions on, 34, pp. 138-153 (2004).

3. Varaiya, P. "Smart cars on smart roads: problems of control", Automatic Control, IEEE Transactions on, 38, pp. 195-207 (1993).

4. Voth, D. "A new generation of military robots", Intelligent Systems, IEEE, 19, pp. 2-3 (2004).

5. Kube, C.R. and Hong, Z. "The use of perceptual cues in multi-robot box-pushing", in Robotics and Automation, Proceedings., 1996 IEEE International Conference on, 3, pp. 2085-2090 (1996).

6. Vidal, T., Ghallab, M. and Alami, R. "Incremental mission allocation to a large team of robots", in Robotics and Automation, Proceedings., 1996 IEEE International Conference on, 2, pp. 1620-1625 (1996).

7. Ailon, A. and Zohar, I. "Control strategies for driving a group of nonholonomic kinematic mobile robots in 
formation along a time-parameterized path", Mechatronics, IEEE/ASME Transactions on, 17, pp. 326336 (2012).

8. Qian, D., Tong, Sh., Guo, J. and Lee, S.G. "Leaderfollower-based formation control of nonholonomic mobile robots with mismatched uncertainties via integral sliding mode", Proceedings of the Institution of Mechanical Engineers, Part I: Journal of Systems and Control Engineering, SAGE Publication (2015). DOI:10.1177/0959651814568365

9. Balch, T. and Arkin, R.C. "Behavior-based formation control for multirobot teams", Robotics and Automation, IEEE Transactions on, pp. 926-939 (1998).

10. Shao, J., Xie, G. and Wang, L. "Leader-following formation control of multiple mobile vehicles", Control Theory \& Applications, IET, 1, pp. 545-552 (2007).

11. Leonard, N.E. and Fiorelli, E. "Virtual leaders, artificial potentials and coordinated control of groups", in Decision and Control, Proceedings of the 40th IEEE Conference on, 3, pp. 2968-2973 (2001).

12. Yangmin, L. and Xin, C. "Dynamic control of multirobot formation", In Mechatronics, ICM '05. IEEE International Conference on, pp. 352-357 (2005).

13. Ghommam, J., Mehrjerdi, H., Saad, M. and Mnif, F. "Formation path following control of unicycle-type mobile robots", Robotics and Autonomous Systems, 58, pp. 727-736 (2010).

14. Dierks, T. and Jagannathan, S. "Control of nonholonomic mobile robot formations: Backstepping kinematics into dynamics", in Control Applications, $C C A$ 2007, IEEE International Conference on, pp. 94-99, (2007).

15. Hassan, G.M., Yahya, K.M. and Haq, I. "Leaderfollower approach using full-State linearization via dynamic feedback", in Emerging Technologies, ICET'06. International Conference on, pp. 297-305 (2006).

16. Freund, E. and Hoyer, H. "Real-time pathfinding in multirobot systems including obstacle avoidance", The International Journal of Robotics Research, pp. 42-70 (1988).

17. Dai, Y., Kim, Y., Wee, S., Lee, D. and Lee, S. "A switching formation strategy for obstacle avoidance of a multi-robot system based on robot priority model", ISA Trans (May 2015). Doi: 10.1016/j.isatra.2014.10.008. Epub 2014 Dec 10.

18. Sayyaadi, H., Kouhi, H. and Salarieh, H. "Control of car-like (wheeled) multi robots for following and hunting a moving target", Scientia Iranica, 18, pp. 950-965 (2011).

19. Greenwood, D.T., Advanced Dynamics, Cambridge University Press, pp. 72-84.

20. Slotine, J.-J.E., Applied Nonlinear Control, Prentice Hall, pp. 208-271 (1991).

21. Gamage, G.W., Mann, G.K.I. and Gosine, R.G. "Leader follower based formation control strategies for nonholonomic mobile robots: Design, implementation and experimental validation", in American Control Conference (ACC), 2010, pp. 224-229 (2010).

\section{Appendix}

$$
\begin{aligned}
& W=1 /\left(-4 I \cos \beta^{2}+8 I \cos \beta^{2} \cos \theta^{2}\right. \\
& +8 I \cos \beta \sin \beta \cos \theta \sin \theta-m d^{2} \cos \phi^{2} \\
& -2 m d^{2} \cos \phi \sin \theta \sin \beta-4 I \cos \theta^{2}-m d^{2} \\
& \left.-2 m d^{2} \cos \theta \cos \phi \cos \beta\right), \\
& g_{21}=-d^{2}(\cos \phi(\cos \theta+\sin \beta \sin \theta \cos \beta \\
& +\sin \beta \sin \theta \cos \phi \cos \theta+\sin \phi \sin \beta \cos ^{2} \theta \\
& -\sin \phi \sin \theta \cos \beta \cos \theta+\cos \beta \cos ^{2} \theta \cos \phi \\
& \left.+\cos ^{2} \beta \cos \theta-\sin \phi \sin \theta \cos ^{2} \beta\right) \\
& +\sin \phi \cos \beta \sin \beta \cos \theta+\cos \beta), \\
& g_{22}=\dot{y}\left(m d^{2} \sin \beta(\cos \phi \sin \theta+\sin \beta)\right. \\
& +4 I \sin \beta \cos \theta(\cos \theta \sin \beta-\cos \beta \sin \theta) \\
& -\dot{x}\left(m d^{2} \cos \beta \sin \theta(1-\cos \phi)\right. \\
& +4 I \cos \beta \cos \theta \sin \phi)), \\
& g_{41}=d^{2}(\cos \phi(-\sin \phi \cos \beta+2 \sin \theta+\cos \beta \sin \beta \cos \theta \\
& -\cos ^{2} \beta \sin \theta+\sin \phi \cos \beta \cos ^{2} \theta \\
& +\sin \phi \sin \beta \cos \theta \sin \theta)+\cos \theta\left(-\sin \phi \cos ^{2} \beta\right. \\
& +\sin \phi)+\sin \beta(1-\sin \phi \sin \theta \cos \beta)), \\
& g_{42}=\dot{x} \cos \beta\left(4 I\left(\cos \beta \cos ^{2} \theta+\sin \theta \sin \beta \cos \theta-\cos \beta\right)\right. \\
& \left.-m d^{2}(\cos \beta-\cos \theta \cos \phi)\right) \\
& +\dot{y} \sin \beta\left(4 I\left(\cos \theta \sin \theta \sin \beta-\cos \beta \sin ^{2} \theta\right)\right. \\
& \left.-m d^{2}(\cos \theta \cos \phi-\cos \beta \sin \beta)\right), \\
& g_{61}=-2 I\left(\operatorname { c o s } ^ { 2 } \beta \left(\sin \phi-2 \sin \phi \cos ^{2} \theta\right.\right. \\
& -2 \sin \theta \cos \theta \cos \phi)+\cos ^{2} \theta(\sin \phi \\
& +2 \cos \beta \sin \beta \cos \phi)+\sin \phi+\cos \theta \sin \theta \cos \phi \\
& -\sin \beta \cos \beta(-2 \sin \phi \sin \theta \cos \theta-\cos \phi)),
\end{aligned}
$$




$$
\begin{aligned}
& g_{62}=-2 m d \dot{x} \cos \beta(\cos \phi+\sin \beta \sin \theta+\cos \beta \cos \theta) \\
& -2 m d \dot{y}\left(\sin \beta(\cos \phi+\cos \theta \cos \beta)+\sin \theta \sin ^{2} \beta\right), \\
& f_{2}=m d^{2} \dot{x} \dot{\theta}(\sin \beta \cos \beta+\cos \phi \sin \beta \cos \theta \\
& \left.+\cos ^{2} \phi \sin \theta \cos \theta+\sin \theta \cos \phi \cos \beta\right) \\
& +4 \operatorname{I} \dot{y} \dot{\theta}\left(-2 \cos ^{2} \theta \cos ^{2} \beta-2 \cos \beta \sin \beta \cos \theta \sin \theta\right. \\
& \left.+\cos ^{2} \theta+\cos ^{2} \beta\right)+m d^{2} \dot{y} \dot{\theta}(2 \cos \phi \sin \beta \sin \theta \\
& \left.-\cos ^{2} \theta \cos ^{2} \phi+\cos ^{2} \phi+\sin ^{2} \beta\right), \\
& f_{4}=-\dot{x} \dot{\theta}\left(m d ^ { 2 } \left(\cos ^{2} \phi \cos ^{2} \theta+2 \cos \theta \cos \phi \cos \beta\right.\right. \\
& \left.+\cos ^{2} \beta\right)+4 I\left(\cos ^{2} \theta-2 \cos ^{2} \beta \cos ^{2} \theta\right. \\
& \left.\left.-2 \sin \beta \sin \theta \cos \beta \cos \theta+\cos ^{2} \beta\right)\right) \\
& -m d^{2} \dot{y} \dot{\theta}\left(\cos \theta \cos \phi \sin \beta+\cos ^{2} \phi \cos \theta \sin \theta\right. \\
& +\cos \phi \cos \beta \sin \theta+\cos \beta \sin \beta),
\end{aligned}
$$

$$
\begin{aligned}
f_{6}= & 2 m d \dot{x} \dot{\theta}\left(-\sin \beta \sin \theta \cos \beta+\cos \theta-\cos ^{2} \beta \cos \theta\right. \\
& +\cos \beta \cos ^{2} \theta \cos \phi-\cos \beta \cos \phi \\
& +\sin \beta \sin \theta \cos \phi \cos \theta)+2 m d \dot{y} \dot{\theta}\left(\sin \theta \cos ^{2} \beta\right. \\
& -\cos \theta \cos \beta \sin \beta-\sin \beta \cos \phi \cos ^{2} \theta \\
& +\cos \beta \cos \theta \cos \phi \sin \theta) .
\end{aligned}
$$

\section{Biography}

Hassan Sayyaadi received his BS degree from Amirkabir University of Technology, Tehran, Iran, in 1987, his MS degree from Sharif University of Technology, Tehran, Iran, in 1990, and his PhD degree from the University of Tokyo, Japan, in 2001, all in Mechanical Engineering. From 1990 to 1996, he was with the Mechanical Engineering Department of Sharif University of Technology and from 2001, after his PhD studies, he has again been with the same department and university. Now, he is an Associate Professor and his research interests include dynamics and control, robotics and mechanisms, artificial intelligence, and neural networks. 